\title{
El DERECHO ANTE LA INTELIGENCIA ARTIFICIAL. A PROPÓSITO DE LO DISPUESTO EN EL ART. 80.5 DEL Convenio Colectivo Nacional de la Banca
}

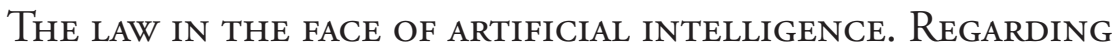 \\ The Provisions of art. 80.5 OF the National Collective \\ Bargaining Agreement for the Banking Sector
}

\author{
Roberto FernándeZ VilLarino \\ Universidad de Huelva \\ Orcid: https://orcid.org/0000-0001-8587-9306 \\ roberto@ddtss.uhu.es
}

Resumen: El proceso de implementación de la inteligencia artificial y los algoritmos en el ámbito de la gestión de los recursos humanos y de las relaciones de trabajo, está generando un profundo debate jurídico en torno a cuestiones tales como la eficiencia de los procesos y decisiones o el grado de intervención humana. También el alcance de la información que recibe el trabajador en este proceso y los riesgos de discriminación derivados del mismo. En el presente estudio, abordaremos dicho debate analizando el alcance del art. 80.5 del Convenio de la Banca, que introduce una regulación de las garantías de los procesos de decisión empresarial basados exclusivamente en la gestión artificial de datos sin la intervención humana. Esta regulación podría suponer un avance en términos de transparencia y gobernanza respecto a la escasa e incompleta regulación específica sobre esta materia existente actualmente, con un elevado predominio de la normativa de softlaw.

Palabras ClaVE: Inteligencia artificial; algoritmo; relaciones laborales; recursos humanos; gobernanza de las relaciones laborales; transparencia; protección de datos

АвSTRACT: The process of implementing artificial intelligence and algorithms in the field of human resources management and labour relations is generating a Recibido: 17-10-2021; Aceptado: 19-10-2021; Versión definitiva: 12-11-2021.

Copyright: (C) Editorial Universidad de Sevilla. Este es un artículo de acceso abierto distribuido bajo los términos de la licencia de uso y distribución Creative Commons Reconocimiento-NoComercialSinObraDerivada 4.0 (CC BY-NC-ND 4.0) 
profound legal debate on issues such as the efficiency of processes and decisions or the degree of human intervention. Also the extent of the information that the employee receives in this process and the risks of discrimination arising from it. In this study, we will address this debate by analysing the scope of Art. 80.5 of the Banking Agreement, which introduces a regulation of the guarantees of business decision-making processes based exclusively on the artificial management of data without human intervention. This regulation could represent an advance in terms of transparency and governance with respect to the scarce and incomplete specific regulation on this matter that currently exists, with a high predominance of softlaw regulations.

KEY wORDs: Artificial intelligence; algorithm; industrial relations; human resources; industrial relations governance; transparency; data protection

SUMARIO: 1. Retos Del Derecho ANTE LA INTELIGENCIA ARTIFICIAL: CONCEPTO Y CONTEXTUALIZACIÓN. 2. LA GOBERNANZA EN LA REGULACIÓN DE LA INTELIGENCIA ARTIFICIAL EN El ÁMbito DE LAS RELACiONES LABORALES. 2.1. La complejidad de regular la IA. 2.2. La verdadera fiabilidad de los resultados formulados por la IA y los algoritmos. 2.3. La participación efectiva de la representación legal de los trabajadores. 3. LA TRANSPARENCIA EN LA REGULACIÓN DE LA INTELIGENCIA ARTIFICIAL EN materia de protección de datos. 4. El art. 80.5 Del Convenio Colectivo NACIONAL DE LA BANCA. 4.1. El riesgo de la discriminación. 4.2. Materialización del riesgo de la discriminación en la utilización concreta de estos sistemas. 5. ConCLUSIONES. 6. Bibliografía.

\section{Retos del Derecho ANTe la inteligencia ARTificial: CONCEPTO Y CONTEXTUALIZACIÓN}

Evitaré comenzar un análisis que aborde el fenómeno de los derechos digitales en el ámbito de las relaciones de trabajo, sin citar conceptos frecuentemente utilizados en las primeras líneas de similares estudios, tales como transformación digital, era de la big data, era de la información, cuarta revolución industrial. Asentada la certeza de estas consideraciones, me parece interesante comenzar evidenciando lo que Innerarity considera como epistemología de la complejidad ${ }^{1}$. Complejidad extrema derivada de la arquitectura del instrumento tecnológico y, por consiguiente, del esfuerzo reflexivo por aspirar a comprenderlo. A partir de su comprensión, poder atisbar las múltiples consecuencias que se derivan de su uso para el desempeño de cualquier acción o actividad que se nos pueda ocurrir y, por ende, sobre las repercusiones que ya está teniendo sobre el ámbito de las relaciones de trabajo. Mencken sostenía que "para todo

$$
\text { 1. } 2020 \text {. }
$$


problema complejo existe una respuesta que es clara, simple y falsa ${ }^{2}$. Por tanto, se sugiere no perder la perspectiva de la complejidad de la materia, porque nos ayudará a entender mejor el status quo de aspectos tales como el grado de desarrollo normativo, el nivel de eficacia de la legislación de protección de datos para las personas trabajadoras, o el enfoque que se le otorga desde la negociación colectiva, entre otras muchas consideraciones.

A nivel conceptual, podemos afirmar que un algoritmo es «un conjunto ordenado y finito de operaciones que permite hallar la solución de un problema», e inteligencia artificial (en adelante: IA) como «aquella disciplina científica que se ocupa de crear programas informáticos que ejecutan operaciones comparables a las que realiza la mente humana, como el aprendizaje o el razonamiento lógico» ${ }^{3}$.

Continuando con las denominaciones y sobre la base conceptual que nos ofrece el artículo 80 del Convenio Colectivo Nacional de la Banca (en adelante Convenio de la Banca), se reconocen como derechos digitales a un conjunto de derechos que dispone la plantilla en el ámbito de las relaciones de trabajo. Así cita como tales: el «derecho a la desconexión digital y laboral» (art. 80.1), el «derecho a la intimidad y al uso de dispositivos digitales en el ámbito laboral» (art. 80.2), el «derecho a la intimidad frente al uso de dispositivos de videovigilancia, grabación de sonidos y geolocalización en el ámbito laboral» (art. 80.3), el «derecho a la educación digital» (art. 80.4), y el «derecho ante la inteligencia artificial» (art. 80.5), que literalmente establece:

"Las nuevas herramientas basadas en algoritmos pueden aportar valor hacia una gestión más eficiente de las Empresas, ofreciendo mejoras en sus sistemas de gestión. Sin embargo, el desarrollo creciente de la aportación de la tecnología requiere de una implantación cuidadosa cuando se aplica en el ámbito de las personas. Por ello, las personas trabajadoras tienen derecho a no ser objeto de decisiones basadas única y exclusivamente en variables automatizadas, salvo en aquellos supuestos previstos por la Ley, así como derecho a la no discriminación en relación con las decisiones y procesos, cuando ambos estén basados únicamente en algoritmos, pudiendo solicitar, en estos supuestos, el concurso e intervención de las personas designadas a tal efecto por la Empresa, en caso de discrepancia.

Las Empresas informarán a la RLT sobre el uso de la analítica de datos o los sistemas de inteligencia artificial cuando los procesos de toma de decisiones en materia de recursos humanos y relaciones laborales se basen, exclusivamente en modelos digitales sin intervención humana. Dicha información, como mínimo, abarcará los datos que nutren los algoritmos, la lógica de funcionamiento y la evaluación de los resultados".

No es casualidad que precisamente sea el Convenio de la Banca, de los primeros en regular esta materia. El uso intensivo de las tecnologías, forma y ha formado parte de la cultura del método de trabajo de la industria financiera. De tal manera que, en
2. 1986.
3. DRAE 2020. 
cada momento histórico se han generado problemáticas y conflictos de derechos, estrechamente vinculadas con el grado de desarrollo del estado de la tecnología. Se ha pasado de las primeras sentencias sobre la privacidad e intimidad de los trabajadores $^{4},-$ muy vinculadas con los límites del uso del software de correo electrónico o visitas de webs- y los derechos de acceso por parte de la empresa al uso laboral de los mismos, a los riesgos derivados de la utilización de la IA y los algoritmos en las relaciones laborales. Así como al modelo de gestión de los recursos humanos, en el que se pueden tomar decisiones sin la intervención humana.

El grado de evolución y complejidad del estado de la tecnología evidencia nuevos conflictos en el ámbito laboral. Hablamos de la vertiginosa e infinita evolución de lo digital (blockchain, redes 5G, internet de las cosas, servicios Cloud, Big data, etc.), pero sobre todo la IA y el uso de los algoritmos, un suceso absolutamente relevante en la historia contemporánea ${ }^{5}$, de consecuencias aún desconocidas. Tecnologías que contribuyen a que las empresas tengan una ventaja competitiva en un mundo hiper-globalizado. Algunos autores hablan de Cuarta Revolución Industrial, poniendo de manifiesto la existencia de una época diferente a la de sus predecesoras, por cuanto implica una cierta disrupción sistémica que afecta tanto a los fundamentos, como al funcionamiento mismo de la vida política, económica y social ${ }^{6}$.

Observamos cómo esta tecnología está pasando de su incorporación al modelo de negocio a trasladarse con fuerza al ámbito de la gestión de los recursos humanos y de las relaciones laborales. En materias que van desde los criterios de selección de personal, modificaciones sustanciales, horarios, organización - planificación-control de trabajo, derecho disciplinario o extinción de las relaciones las relaciones laborales entre otros ${ }^{7}$. Por otra parte, tal y como sostiene cierta doctrina, en su proceso de implementación existen elementos condicionantes para la empresa, muy vinculados a elementos tales como la afectación de las condiciones de trabajo básicas, las relacionadas con la determinación del objeto de la prestación, el tiempo de trabajo y la

4. Entre otros muchos, el tan comentado asunto Deutsche Bank (marzo de 2001) sobre el uso privado del email de la empresa.

5. Stephen Hawking viene afirmando que "Artificial Intelligence could be the biggest event in the history of our civilisation. Or the worst. We just don't know". Sobre esta cuestión puede igualmente consultarse Robles Carrillo 2020a.

6. Robles Carrillo 2020b, consultado online.

7. En este mismo sentido, Mercader Uguina 2021, 5: “... la empresa la que ha incorporado su uso hasta el punto de que parece que el empresario está dispuesto a delegar o, si se prefiere, a descentralizar parte de sus poderes tradicionales trasladando un importante número de decisiones a la presunta objetividad y plena fiabilidad que proporciona el recurso al Big Data. Y ello en la medida en que su uso actual se proyecta sobre prácticamente la totalidad de las facetas que componen su autonomía organizativa, recorriendo transversalmente la libertad de actuación empresarial, abarcando desde la selección de trabajadores hasta la forma y modo de ejercicio del poder de dirección, incluyendo sus dimensiones disciplinarias". 
forma y criterios de remuneración ${ }^{8}$. Pero también a la nueva consideración del trabajo requerido que vendrá condicionado por las tareas a desempeñar ${ }^{9}$; resultando este concepto, el de tareas, el que puede adquirir centralidad en la definición del contenido y el objeto de la prestación. Entre los factores que han contribuido a esta vertiginosa evolución, por la doctrina se apunta al escaso coste de implantación de estas tecnologías en relación con su elevado margen de eficiencia en los procesos en los que se ha incorporado ${ }^{10}$.

En todo caso, considero la cuestión relacionada con el ámbito de la gobernanza de las relaciones de trabajo, como un vector especialmente interesante en la implementación de la IA. Entendiendo por gobernanza la metodología por la que se toman decisiones que afectan a la esfera de derechos individuales de las personas trabajadoras. En efecto, cuando hablamos de que forman parte del ámbito de decisión, estamos considerando la propia gobernanza de la organización. Circunstancia que, como veremos multiplica las consideraciones, reflexiones o debates, no sólo empresariales o éticas sino fundamentalmente jurídicas.

Así, la gobernanza de la IA está planteando diversos problemas de naturaleza conceptual (qué es exactamente la IA y algoritmos), funcional (cómo funcionan), de orden práctico (todo lo que respecta a su implementación) y, especialmente, de naturaleza jurídica (relaciones entre la aplicación de la IA al derecho y la regulación de la IA por el derecho $)^{11}$. En paralelo la materia está ocupando un mayor espacio de atención en todo cuanto nos rodea: desde nuestros hábitos de consumo, pasando por el ocio y el entretenimiento, hasta el ámbito laboral que estamos analizando.

Se evidencia un vasto y complejo debate en el que identificamos un problema principal que posiblemente, sea la causa fundamental de buena parte de la controversia existente en torno al tema: no hay un lenguaje común, ni una metodología única o realmente interdisciplinar a la hora de enfocar el propio debate, tratamiento y alcance del fenómeno ${ }^{12}$. Por tanto, la organización de la gobernanza de la IA se hace aún más complicada por el hecho de no existir un concepto consensuado y aceptado, formulado jurídicamente, para dotarlo de mayor seguridad y garantías, sobre el que operar para garantizar la viabilidad misma de la discusión y sus resultados.

8. Valverde Asencio 2020, 45.

9. Directamente relacionado con la consideración del trabajo en torno a tareas y dificultades de definir la amplitud de su incidencia: Evans-Freenwood, Lewis, Guszcza 2017.

10. Todolí Signes 2018, consultado online.

11. Las transformaciones que implican las TIC alientan la superación de la idea de gobierno y la transición hacia modelos de gobernanza: Becerra, et al. 2018, 19 y ss.

12. Robles Carrillo 2020b, consultado online: "A este problema conceptual se suma otro de orden metodológico y funcional. La polarización del debate entre la técnica y la ética está limitando y tergiversando los términos del mismo y sus resultados. La falta de comprensión que de ello se deriva limita las posibilidades de avanzar de un modo real y fructífero en la organización y regulación de la IA". 
El elemento nuclear de la formulación jurídica del proceso de implementación de estas tecnologías parte del modelo de toma de decisiones en el ámbito laboral, esto es, de como a través de la gobernanza se puede mitigar los eventuales impactos negativos del uso de los algoritmos y la IA sobre los derechos fundamentales de las personas trabajadoras. El tratamiento y abordaje completo de este proceso enlaza necesariamente con la consideración del modelo o sistema de gobernanza de las relaciones laborales y el papel que ocupa la transparencia, como elemento principal del deber de buena fe, del que es absoluto titular el empresario. Esto es, de cómo el grado de implantación en todas las aristas derivadas de la utilización de estos sistemas (desde el vector tecnológico al jurídico) debe ser absoluta y completamente transparente. Finalmente enlaza también con el concepto de la gobernanza de la protección de datos en el ámbito laboral y del rol que ostenta y puede ostentar en ella, la representación legal de los trabajadores ${ }^{13}$.

A su vez podemos afirmar que la cuestión de la gobernanza en los derechos derivados de la IA y algoritmos, está siendo tratada (aún) como iniciativa de soft-law tanto a nivel europeo como nacional. En este sentido, siendo como es el asunto nuclear y ocupando un lugar más que relevante en la agenda política europea, observamos sin embargo un evidente grado de inmadurez a nivel de propuestas normativas concretas.

La consideración de estos elementos, constituirán los elementos de análisis y debate que pretendemos considerar a lo largo del presente estudio, por cuanto una lectura atenta del art. 80.5 del Convenio de la Banca, nos conduce indefectiblemente a ella. Sobre la base de este objetivo, debemos contemplar el marco normativo vigente, junto con determinadas iniciativas legales, que apuntan al modelo de gobernanza y transparencia con elementos comunes que van, desde la identificación y consideración del riesgo a la discriminación, al contenido concreto de los derechos de los trabajadores ${ }^{14}$.

\section{LA GOBERNANZA EN LA REGULACIÓN DE LA INTELIGENCIA ARTIFICIAL EN EL ÁMBITO DE LAS RELACIONES LABORALES}

A nivel europeo, destacamos una serie de iniciativas y propuestas que constituyen buen ejemplo de que la IA se regulará desde la perspectiva del respeto a los valores y

13. Forma igualmente parte de esta consideración de gobernanza, el debate entre los derechos específicos -granulares como los denomina Mercader (2021)- frente a la necesidad de considerar a la representación legal de los trabajadores dentro del ámbito de la gobernanza en esta materia. Superando con ello el carácter o naturaleza individual de la legislación de protección de datos.

14. En especial, conocer con detalle los elementos sobre los que se han tomado decisiones que les conciernen. 
derechos fundamentales de la persona. A su vez, todas ellas incluyen el común denominador de garantizar que se promueva la innovación a la junto con el respeto a los principios éticos, la transparencia y la obligación de rendir cuentas. Se trata de la Estrategia Europea en IA de 25/4/2018, el Plan Coordinado de 7/12/2018, las Directrices éticas sobre IA fiable de abril de 2019, la Resolución Parlamento Europeo de 12/2/2019 y el Libro Blanco sobre IA de 19/02/2020.

Por otra parte, la Agencia Europea de los Derechos Fundamentales ha elaborado una propuesta de protección de los derechos fundamentales en el desarrollo y uso de algoritmos. Dos son las líneas de actuación que se contemplan para ello, por una parte, la exigencia de transparencia sobre el proceso de elaboración de los algoritmos: desde el desarrollo de estas herramientas, hasta la consideración, detección y rectificación de cualquier error en su aplicación. Por otra parte, tal como apunta la doctrina ${ }^{15}$, se aboga por la realización de una evaluación del impacto de los derechos fundamentales, para identificar posibles sesgos y abusos en la aplicación y en los resultados obtenidos por los algoritmos. Esta evaluación incluye, entre otras, una medición del "potencial de discriminación», en relación con motivos diferentes, como género, edad, etnia, religión, orientación sexual y política ${ }^{16}$.

Por lo que respecta al ámbito nacional español y, directamente vinculado con este debate público institucional, citamos el documento de trabajo Las decisiones algoritmicas en las relaciones laborales, instado por la Unión General de los Trabajadores y que propugna una regulación precisa que obligue entre otras cuestiones a dar a conocer cuáles son los criterios que maneja la $\mathrm{IA}^{17}$. El documento denomina a este proyecto de propuesta normativa como la potencial «Ley de Justicia algorítmica en las relaciones laborales».

Por otra parte, la Carta de Derechos Digitales adoptada por el Gobierno de España $^{18}$, de 15 de julio de 2021. Se trata de un documento que, sin tener carácter normativo, ofrece el primer marco de referencia para garantizar los derechos de la ciudadanía en la nueva realidad digital y tiene como objetivo reconocer los retos que plantea la adaptación de los derechos actuales al entorno virtual y digital. El texto recoge un conjunto de principios y derechos para guiar futuros proyectos normativos y el desarrollo de las políticas públicas de forma que se garantice la protección de los derechos individuales y colectivos en los nuevos escenarios digitales.

Al hilo de la Carta de Derechos Digitales, destaca la anunciada creación de un Observatorio Social de los algoritmos, por parte del Gobierno de España (a través de

15. Sáez Lara 2020, 57.

16. Al mismo tiempo, las evaluaciones de impacto podrían evaluar el posible sesgo de discriminación derivado de usar "proxy" información, como por ejemplo puede ser el lugar de residencia, con respecto a motivos protegidos, raza, en el área de discriminación. Vid. Informe FRA 2018.

17. Documento UGT 2021.

18. Gobierno de España 2021. 
la Secretaría de Estado de Digitalización e Inteligencia Artificial) ${ }^{19}$. Precisamente tendrá como objetivo la vigilancia de los Algoritmos para que no incurran en discriminaciones por su programación.

Todas estas iniciativas, están impulsando una interesante bibliografía sobre el propio concepto de gobernanza, a tener en cuenta si queremos mantener un riguroso debate en torno al alcance del equilibrio que hemos venido subrayando en este análisis: eficacia de estos sistemas sobre el respeto a los derechos fundamentales de las personas trabajadoras. Así reflexionamos a continuación sobre la necesidad de analizar y fijar los conceptos básicos del debate sobre la gobernanza en cada uno de los elementos que se exponen a continuación.

\subsection{La complejidad de regular la IA}

La gobernanza de la IA constituye, posiblemente, uno de los mayores desafíos que se ha planteado a la ciencia y a la técnica jurídicas. Tal como indica la doctrina, material y teleológicamente, la aparición de esta tecnología altera los parámetros básicos de la organización humana y social, incluso cuando aún no se ha manifestado en toda su potencialidad, desde el momento en que apunta hacia la posibilidad de emular o perfeccionar al ser humano ${ }^{20}$.

Esta complejidad se materializa en la práctica que, tal como hemos observado la mayoría de los Estados aún no ha desarrollado estrategias o planes de gobernanza. En el plano internacional, la acción de Naciones Unidas es muy amplia en asuntos sectoriales, pero no avanza en la definición del modelo global de gobernanza ${ }^{21}$.

\subsection{La verdadera fiabilidad de los resultados formulados por la IA y los algoritmos}

A menudo surgen dudas sobre cómo se han formulado los resultados concretos de estos procesos y si efectivamente pueden quedar espacios dentro del sistema analítico del algoritmo de difícil acceso. Tal y como apunta la doctrina ${ }^{22}$, transparencia y evaluación deben de verificarse tanto sobre la calidad de los datos, como sobre la forma en que funcionan los algoritmos (verificable en una caja negra del algoritmo), así como el alcance de las facultades predictivas de los mismos. La evaluación de los algoritmos, desde la perspectiva de los derechos fundamentales, es percibida como una tarea compleja que exige de una evaluación global, que incluya los efectos del

19. Anuncio recogido por varios medios de comunicación en fecha 21.07.21, entre otros Europa Press, https://www.europapress.es/economia/noticia-gobierno-creara-observatorio-social-algoritmos-20210721134837.html.

20. Robles Carrillo 2020b, consultado online.

21. Robles Carrillo 2020b, consultado online.

22. Robles Carrillo 2020a, consultado online. 
uso de los datos sobre las libertades y derechos fundamentales ${ }^{23}$. Su necesidad está completamente justificada. Si se pretende obtener una eficaz protección de los derechos fundamentales frente a la IA, es preciso articular herramientas que garanticen las debidas exigencias de transparencia y explicabilidad ${ }^{24}$, así como una evaluación amplia del impacto sobre los derechos fundamentales. Estas exigencias serían las herramientas a esgrimir frente a las eventuales discriminaciones algorítmicas ${ }^{25}$.

\subsection{La participación efectiva de la representación legal de los trabajadores}

Sin duda el elemento que resulta absolutamente clave en el proceso de implementación de la IA tanto en materia de selección de personal, como en todas las variantes del poder de dirección de la empresa ${ }^{26}$. Además, configuraría un verdadero y eficaz modelo de gobernanza. Una auténtica gobernanza colectiva de la protección de datos, conforme al mandato contemplado en el art. 88.1 del Reglamento (UE) 2016/679 del Parlamento Europeo y del Consejo, relativo a la protección de las personas físicas en lo que respecta al tratamiento de datos personales y a la libre circulación de estos datos y por el que se deroga la Directiva 95/46/CE (en adelante: RGPD) ${ }^{27}$ :

23. Como el ya referido principio de no discriminación; en este sentido: Mantelero 2018, 772.

24. En este mismo sentido, Robles Carrillo 2020b, consultado online: "Para hacer realidad una IA fiable, centrada en la persona y en el respeto de los derechos fundamentales, será necesario establecer exigencias de transparencia, explicabilidad y rendición de cuentas o "responsabilidad algorítmica". Gracias a la transparencia y explicabilidad deberán identificarse y podrán eliminarse los sesgos discriminatorios, en la fase de recopilación de la información o en los propios métodos de desarrollo del sistema de IA (por ejemplo, la programación del algoritmo). Pero será necesario complementar los requisitos anteriores con la rendición de cuentas, que exige establecer mecanismos que garanticen la responsabilidad de los sistemas de IA y de sus resultados. A estos efectos, sería necesaria la fijación de auditorías independientes, que evalúen los algoritmos (los datos y los procesos de diseńo)".

25. Sáez Lara 2020, 58. "En las propuestas analizadas (se refiere a las distintas iniciativas europeas de regulación de la materia), se aprecia un elemento común; la consideración de la discriminación algorítmica como un riesgo frente al que ha de establecerse un marco normativo de tutela preventiva. Más concretamente, centrando su aplicación al ámbito de la empresa y a su actuación en el terreno de la decisión empresarial, esta tutela preventiva frente al riesgo de la discriminación algorítmica impondría al empresario una evaluación de impacto o una auditoría interna para medir y corregir, en su caso, el impacto discriminatorio de las decisiones automatizadas". Sigue sosteniendo que "sobre la base de todo lo anterior, la propuesta para la regulación espańola nos llevaría a introducir en nuestra normativa laboral la obligación legal del empresario de evaluar el impacto discriminatorio de las decisiones automatizadas incluida la elaboración de perfiles, con efectos sobre las personas trabajadoras, en el empleo y en las condiciones de trabajo, a través de una reforma del art. 17 LET".

26. Entre otros muchos, Valverde Asencio 2020, 112-119.

27. De 27 de abril de 2016. 
"Los Estados miembros podrán, a través de disposiciones legislativas o de convenios colectivos, establecer normas más específicas para garantizar la protección de los derechos y libertades en relación con el tratamiento de datos personales de los trabajadores en el ámbito laboral, en particular a efectos de contratación de personal, ejecución del contrato laboral, incluido el cumplimiento de las obligaciones establecidas por la ley o por el convenio colectivo, gestión, planificación y organización del trabajo, igualdad y diversidad en el lugar de trabajo, salud y seguridad en el trabajo, protección de los bienes de empleados o clientes, así como a efectos del ejercicio y disfrute, individual o colectivo, de los derechos y prestaciones relacionados con el empleo y a efectos de la extinción de la relación laboral".

Se trata de incorporar el derecho de que la representación legal de los trabajadores pueda negociar (o al menos ser consultados) en esta materia, que tengan la prerrogativa no solo de ejercer ciertos derechos en nombre de los trabajadores, sino también de tener la posibilidad de verificar el uso que se realiza de la información y datos de las personas trabajadoras y de controlar que la información utilizada se ha obtenido y procesado de manera lícita, lo que se acercaría al ámbito de la gobernanza de la protección de datos, tal y como considera un sector de la doctrina ${ }^{28}$.

\section{LA TRANSPARENCIA EN LA REGULACIÓN DE LA INTELIGENCIA ARTIFICIAL EN MATERIA DE PROTECCIÓN DE DATOS}

Si al analizar el vector gobernanza en la regulación de la IA en las relaciones de trabajo estábamos ante lo que podríamos denominar iniciativas de soft-law, en el ámbito del análisis del vector transparencia, se evidencia un incremento del tratamiento normativo aplicado. Así, en el ámbito europeo, destacamos la Directiva (UE) 2019/1152 del Parlamento Europeo y del Consejo de 20 de junio de 2019 relativa a unas condiciones laborales transparentes y previsibles en la Unión Europea, que obliga a informar al trabajador acerca de la retribución inicial de base, así como de "cualquier otro componente, en su caso, indicados de forma separada", de su periodicidad, y del «método de pago», el primer día de trabajo o, a más tardar, el séptimo día natural (art. 4.2.k) ${ }^{29}$.

Antes de esta norma, ya estaba vigente la Directiva 2002/14/CE del Parlamento Europeo y del Consejo, de 11 de marzo de 2002, por la que se establece un marco general relativo a la información y a la consulta de los trabajadores en la Comunidad

28. Todolí Signes 2018, 16.

29. Aunque el soporte adecuado de dicha información sea el contrato de trabajo que, cuando menos, debería delimitar las prestaciones que constituyen su objeto mismo, la Directiva admite su transmisión "en forma de uno o más documentos" (art. 5), e incluso mediante una remisión a las disposiciones legales, reglamentarias, o convencionales reguladoras de la materia. 
Europea. A tenor de la misma, ostentarán derecho a ser informados acerca de «las decisiones que pudieran provocar cambios sustanciales en cuanto a la organización del trabajo y a los contratos de trabajo», (art. 4.2.c) «en un momento, de una manera y con un contenido apropiados» (art. 4.3), gozando cada Estado miembro de libertad para escoger el medio que estime oportuno.

El marco de los aspectos éticos de la inteligencia artificial, la robótica y las tecnologías conexas ${ }^{30}$, ha recordado «la necesidad de consultar e informar a los trabajadores y sus representantes a la hora de introducir sistemas de IA que pudieran provocar cambios en la organización del trabajo, la vigilancia y su control, así como en los sistemas de evaluación y contratación de los trabajadores».

En el ámbito de la legislación laboral interna, la novedad más relevante es la establecida por el Real Decreto-ley 9/2021, de 11 de mayo, por el que se modifica el texto refundido de la Ley del Estatuto de los Trabajadores, aprobado por el Real Decreto Legislativo 2/2015, de 23 de octubre, más comúnmente conocido como Ley Ryder, se aprueba con el objetivo de garantizar los derechos laborales de las personas dedicadas al reparto en el ámbito de plataformas digitales. Resultando el nuevo apartado al art. 64.4 d) en el E.T. que determina el derecho de información al Comité de Empresa de

“d) Ser informado por la empresa de los parámetros, reglas e instrucciones en los que se basan los algoritmos o sistemas de inteligencia artificial que afectan a la toma de decisiones que pueden incidir en las condiciones de trabajo, el acceso y mantenimiento del empleo, incluida la elaboración de perfiles".

Más allá de las fuentes de derechos propias de la relación laboral, el supuesto de hecho de la materia se expande de manera natural, al ámbito de la regulación de la protección de datos. Datos que nutren a los sistemas de IA y, a partir de los cuales, se diseña la arquitectura de las decisiones que conciernen a las personas trabajadoras. En este sentido, el art. 22.1 RGPD, establece una prohibición general de las decisiones basadas únicamente en el tratamiento automatizado, así «Todo interesado tendrá derecho a no ser objeto de una decisión basada únicamente en el tratamiento automatizado, incluida la elaboración de perfiles, que produzca efectos jurídicos en él o le afecte significativamente de modo similar». Se entiende por tal, conforme a lo dispuesto en el art. 4.4 del RGPD,

30. Resolución del Parlamento Europeo, de 20 de octubre de 2020, con recomendaciones destinadas a la Comisión sobre un marco de los aspectos éticos de la inteligencia artificial, la robótica y las tecnologías conexas (2020/2012(INL)), Acceso a documento completo (15.10.21).

https://eur-lex.europa.eu/legal-content/ES/TXT/HTML/?uri=CELEX:52020IP0275\&from=EL 
"toda forma de tratamiento automatizado de datos personales consistente en utilizar datos personales para evaluar determinados aspectos personales de una persona física, en particular para analizar o predecir aspectos relativos al rendimiento profesional, situación económica, salud, preferencias personales, intereses, fiabilidad, comportamiento, ubicación o movimientos de dicha persona física”.

No obstante, el segundo apartado del artículo 22, establece excepciones a esta norma de gran impacto en el ámbito laboral. Así, (lo dispuesto en el) «apartado 1 no se aplicará si la decisión: a) es necesaria para la celebración o la ejecución de un contrato entre el interesado y un responsable del tratamiento"; o bien: «c) se basa en el consentimiento explícito del interesado». Como observamos, estas excepciones dejan un amplio espacio a la implementación de las técnicas y métodos de IA sin intervención humana, en el ámbito de la toma de decisiones en la gestión laboral ordinaria de las empresas en general y muy particularmente, en el ámbito de la toma de decisiones en materia de recursos humanos.

Es por ello que, en aras de proteger el espacio de derechos de las personas trabajadoras y, de prevenir los eventuales riesgos inherentes al uso de estos sistemas que, más adelante desgranaremos, el Considerando 63 RGPD establece que

"todo interesado debe, por tanto, tener el derecho a conocer y a que se le comuniquen, en particular, los fines para los que se tratan los datos personales, su plazo de tratamiento, sus destinatarios, la lógica implícita en todo tratamiento automático de datos personales y, por lo menos cuando se base en la elaboración de perfiles, las consecuencias de dicho tratamiento".

Así, el art. 22.3 del mismo texto legal, determina un conjunto de garantías que la empresa debe cumplir "para salvaguarda de los derechos y libertades y los intereses legítimos del interesado", siendo estas garantías, "como mínimo el derecho a obtener intervención humana por parte del responsable, a expresa su punto de vista y a impugnar la decisión».

La doctrina ha venido interpretando ${ }^{31}$, conforme a este artículo, que existe una obligación del responsable de datos de informar al afectado de las razones que han llevado a tomar esa decisión. Es decir, la empresa, cuando tome decisiones automatizadas, deberá indicarlo e informar de qué parámetros ha utilizado para alcanzar dicha resolución ${ }^{32}$. Esta interpretación está apoyada por el art. 5 RGPD que exige que el procesamiento de datos sea legal, justo y transparente ${ }^{33}$. También, por el

31. Entre otros, Todolí Signés 2018.

32. Y qué ponderación ha asignado a cada uno de ellos.

33. Así, (los) "Principios relativos al tratamiento 1. Los datos personales serán: a) tratados de manera lícita, leal y transparente en relación con el interesado («licitud, lealtad y transparencia»)”. 
artículo $13.2 \mathrm{f})^{34}$ y por lo dispuesto en el art. $14.2 \mathrm{~g}$ ) RGPD, que exige que cuando el sujeto es objeto de decisiones automatizadas, incluyendo la elaboración de perfiles, el responsable de datos deberá entregar al sujeto, información significativa sobre la lógica aplicada, así como la importancia y las consecuencias previstas de dicho tratamiento para el interesado. Ello para garantizar un tratamiento de datos leal y transparente respecto del interesado.

Por tanto, los artículos 13.2.f) y 14.2.g) del RGPD configuran lo que alguna autora ha denominado como el derecho de explicación sobre la lógica aplicada por las decisiones exclusivamente automatizadas (así como sobre la importancia y las consecuencias previstas de dicho tratamiento para el interesado), que deberá realizarse a través de una información específica y de fácil acceso ${ }^{35}$. El RGPD exige que el responsable del tratamiento ofrezca información significativa sobre la lógica aplicada; no necesariamente una compleja explicación de los algoritmos utilizados o la revelación de todo el algoritmo, pero si una información suficientemente exhaustiva para que el interesado entienda los motivos de la decisión ${ }^{36}$. Con ello se pretenden evitar sesgos del algoritmo que no puedan ser controlados por el interesado, precisamente por desconocer las razones por las que se has tomado dicha decisión. Como sostiene la doctrina, el grado de explicación dada por la empresa sobre las razones de la toma de esa decisión deben ser las suficientes para que el interesado pueda ejercer sus derechos a expresar su punto de vista y a impugnar la decisión ${ }^{37}$.

Pero, procede plantearnos qué efectividad tendría este derecho a la explicación, en la lógica que utilizan los algoritmos, y si pudieran existir espacios invisibles o protegidos por los diseñadores de su arquitectura con el pretendido o no, objetivo de

34. Que establece: «2. Además de la información mencionada en el apartado 1, el responsable del tratamiento facilitará al interesado, en el momento en que se obtengan los datos personales, la siguiente información necesaria para garantizar un tratamiento de datos leal y transparente: f) la existencia de decisiones automatizas, incluida la elaboración de perfiles, a que se refiere el artículo 22, apartados 1 y 4, y, al menos en tales casos, información significativa sobre la lógica aplicada, así como la importancia y las consecuencias previstas de dicho tratamiento para el interesado».

35. Sáez Lara 2020, 55.

36. En sus recomendaciones, el citado GT29 indicaba que, en lugar de ofrecer una compleja explicación matemática sobre cómo funcionan los algoritmos o el aprendizaje automático, el responsable del tratamiento debe considerar la utilización de formas claras y exhaustivas de ofrecer información al interesado, por ejemplo: las categorías de datos que se han utilizado o se utilizarán en la elaboración de perfiles o el proceso de toma de decisiones; por qué estas categorías se consideran pertinentes; cómo se elaboran los perfiles utilizados en el proceso de decisiones automatizadas, incluidas las estadísticas utilizadas en el análisis; por qué este perfil es pertinente para el citado proceso; y cómo se utiliza para una decisión relativa al interesado. Vid. Grupo de Trabajo sobre protección de datos del art. 29, Directrices sobre decisiones individuales automatizadas y elaboración de perfiles a los efectos del Reglamento 2016/679. (Acceso a documento completo 25.10.21) https:/www.aepd.es/sites/default/files/2019-12/ wp251rev01-es.pdf.

37. Todolí Signes 2018, 11. 
complicar la comprensión de su funcionamiento. En este sentido, la doctrina sostiene que no puede desconocerse que la efectividad de este derecho de explicación ha sido puesta en duda por quienes afirman que los algoritmos contemporáneos se basan en modelos, que exhiben una lógica implícita, más que explícita, por lo que la lógica subyacente a su toma de decisiones ${ }^{38}$. Algunos autores denominan a estos espacios una caja negra, ininterpretable ${ }^{39}$. Por ello, se concluye que el derecho de explicación se comprende mejor a la luz de los citados preceptos, que reconocen a la autoridad de control poderes de investigación y sanción necesarios para garantizar la efectividad del derecho a la explicación de las personas afectadas por decisiones automatizadas ${ }^{40}$.

En definitiva, si estamos ante decisiones únicamente automatizadas (aunque sean parciales), habrá de garantizarse al interesado, el derecho a obtener una intervención humana por parte del responsable, a expresar su punto de vista y a impugnar su decisión. Y, finalmente, la empresa estaría bajo los poderes de investigación y sanción de las autoridades de control de protección de datos, entre nosotros de la Agencia Española de Protección de Datos. En esta correlación de derechos individuales y de poderes o competencias de la autoridad de control se encontraría la garantía de la efectividad del derecho de explicación de las personas afectadas por decisiones automatizadas.

\section{El art. 80.5 del Convenio Colectivo Nacional de la Banca}

A partir del análisis de las premisas anteriores, vamos a tratar de perfilar el contexto jurídico que rodea el fenómeno de la IA y los algoritmos, en el ámbito del contenido de lo dispuesto en el art. 80.5 del Convenio de la Banca.

Este art. 80.5 recoge dos variantes en torno al alcance de lo que considera el derecho ante la inteligencia artificial: 1.- (...) «a no ser objeto de decisiones basadas única y exclusivamente en variables automatizadas, salvo en aquellos supuestos previstos por la Ley», y 2.- (...) «derecho a la no discriminación en relación con las decisiones y procesos, cuando ambos estén basados únicamente en algoritmos, pudiendo solicitar, en estos supuestos, el concurso e intervención de las personas designadas a tal efecto por la Empresa, en caso de discrepancia».

38. Sáez Lara 2020, 55.

39. Goodman, Flaxman 2016; Wachter, Mittelstadt, Floridi 2017, 76-99.

40. Téngase en cuenta que las multas previstas por el incumplimiento de esta obligación de informar sobre la lógica de la decisión automatizada pueden alcanzar hasta los 20 millones de euros o el 4\% del volumen de negocio anual del ejercicio anterior, optándose por la de mayor cuantía, lo que determina que ninguna empresa sea inmune al cumplimiento. Vid. Casey 2018, 42. 
Frente a esta regulación el trabajador tendría como salvaguarda para evitar el riesgo a no ser discriminado o verse afectado por decisiones basadas exclusivamente en variables automatizadas, a recibir información (rendición de cuentas) de cómo ha funcionado el proceso y a solicitar que intervengan personas designadas por la empresa. En este sentido, sigue diciendo el art. 80 (in fine),

"las Empresas informarán a la RLT sobre el uso de la analítica de datos o los sistemas de inteligencia artificial cuando los procesos de toma de decisiones en materia de recursos humanos y relaciones laborales se basen, exclusivamente en modelos digitales sin intervención humana. Dicha información, como mínimo, abarcará los datos que nutren los algoritmos, la lógica de funcionamiento y la evaluación de los resultados".

El propio art. 80 comienza citando la eficiencia y utilidad de estas herramientas basadas en algoritmos si bien acentúa la necesidad de implementarlas con precaución. Así, el art. 80 recoge que

"las nuevas herramientas basadas en algoritmos pueden aportar valor hacia una gestión más eficiente de las Empresas, ofreciendo mejoras en sus sistemas de gestión. Sin embargo, el desarrollo creciente de la aportación de la tecnología requiere de una implantación cuidadosa cuando se aplica en el ámbito de las personas".

Procede la revisión crítica en torno a si esta regulación resulta eficaz de cara a equilibrar, el uso de la misma por parte de la empresa con los derechos fundamentales de las personas trabajadoras. Esto es, si la transparencia y el derecho a la explicación de la que es deudor el empresario, son elementos suficientes para mitigar el riesgo de discriminación que se trata de evitar. Todo ello sobre la consideración de qué modelo de gobernanza de las relaciones de trabajo plantea este artículo 80.5, en relación con lo establecido por otros convenios que están comenzando a contemplar la regulación de estos fenómenos. Elemento que considero de especial interés en el estado actual de la utilización aún incipiente de los sistemas de IA y algoritmos.

En este sentido, el III Acuerdo para el Empleo y la Negociación Colectiva 2015, 2016 y $2017^{41}$, prorrogado por el IV Acuerdo para el Empleo y la Negociación Colectiva ${ }^{42}$, con el objeto de impulsar el empleo de calidad y con derechos, propugna que los convenios colectivos contemplen, entre otros objetivos fundamentales, la incidencia de las tecnologías de la información y de la comunicación en el desarrollo productivo general y en las relaciones laborales y en materia de seguridad y salud en el trabajo.

41. BOE de 20 de junio de 2015.

42. BOE de 18 de julio de 2018. 
El convenio colectivo de grandes almacenes (21/22), en su Disposición Transitoria Undécima., aborda los compromisos para el gobierno de la transición digital y organizativa del sector. En su apartado 1) establece la

"creación del Observatorio sectorial: Como foro estable de diálogo social y concertación colectiva sobre aquellas materias de interés común, se crea un Observatorio Sectorial desde el que los interlocutores sociales firmantes del presente Convenio realizarán los correspondientes análisis conjuntos de la realidad sectorial, de los cambios que la digitalización y las tendencias de consumo produzcan en la misma, de la competitividad y su evolución, del posicionamiento de las empresas en el mercado, la mejora de las condiciones laborales y de la calidad en el empleo, la formación y la igualdad de oportunidades a partir de la diversidad de entidades incluidas en su ámbito de aplicación”.

En su apartado 2) determina que

"el Observatorio sectorial podrá remitir al ámbito de cada empresa o grupo de empresa el establecimiento de Protocolos para la transición digital y los cambios organizativos, conectados con los objetivos y funciones del Observatorio sectorial, pudiendo a su vez prever la articulación de marcos innovadores de diálogo social («SandBox», observatorios o laboratorios). En dichos Protocolos se podrán definir planes de formación enfocados a la adaptación a los cambios funcionales, competencias digitales y empleabilidad de las plantillas en los nuevos empleos que puedan crearse, dicha formación será adecuada y permanente para el uso de la tecnología, siguiendo las líneas establecidas en la comisión paritaria sectorial del convenio colectivo de grandes almacenes".

Finalmente zanja la mención a la utilización de los algoritmos en el ámbito laboral con un parco "(el) Observatorio prestará especial atención a la utilización de algoritmos que incidan en las condiciones de trabajo".

En términos muy similares al Convenio de la banca, se pronuncia el Convenio actualizado de la construcción en su art. 29.3 ${ }^{43}$. Literalmente regula que

"la implantación de cualquier sistema por parte de la empresa de tecnologías de la información deberá respetar en todo momento la normativa de protección de datos, y en concreto el Reglamento (UE) 2016/679 del Parlamento Europeo y del Consejo, de 27 de abril de 2016, relativo a la protección de las personas físicas en lo que respecta al tratamiento de datos personales y a la libre circulación de estos datos y de la Ley Orgánica 3/2018, de 5 de diciembre, de Protección de Datos Personales y garantía de los derechos digitales, así como cualquier otra normativa que resulte de aplicación o que la sustituya”.

43. Resolución de 23 de abril de 2019, de la Dirección General de Trabajo, por la que se registra y publica el Acta por la que se modifica el Convenio colectivo general del sector de la construcción. BOE 29.04.2019. 
Sigue diciendo:

"En cualquier caso, las medidas que se adopten deben ser proporcionales a la finalidad que tengan, respetando la dignidad y el derecho a la protección de datos, teniendo en cuenta no obstante que ya que deriva de la existencia de una relación laboral no se requiere consentimiento del trabajador. Deberá en todo caso cumplirse con los deberes de información previa a los trabajadores afectados que se establecen en la legislación vigente. Asimismo, cuando estas medidas tengan carácter colectivo o plural deberá informarse previamente a su implantación a los representantes de los trabajadores, indicando la finalidad que se persigue".

\subsection{El riesgo de la discriminación}

Observamos como estos primeros convenios colectivos consideran que estamos ante una realidad que mejorará la eficacia de los procedimientos de selección de personal y por otra, que optimizará la toma de decisiones en gran parte de los aspectos de las relaciones de trabajo. En el que los riesgos (que ahora trataremos) son evidentes y casi exclusivamente derivados de la discriminación y que para evitarlos se fía, así todo al que hemos denominado como derecho a la explicación (con las dudas derivadas del diseño de la propia tecnología que hemos considerado). En función de esa claridad de la explicación, las personas trabajadoras afectadas por las decisiones de la IA o el algoritmo, tendrían posibilidades de plantear reclamaciones, o lo que es lo mismo, poder disponer de un pleno derecho a su tutela judicial efectiva, art. 24 de la Constitución. Todo ello considerando, que estamos hablando del nuevo modelo de gobernanza de las relaciones laborales, en el que debe primar el principio de la buena fe, por parte del empresario a la hora de implementar estos sistemas en el ámbito del desarrollo del ejercito del poder de dirección empresarial cuya titularidad ostenta conforme al art. 38 de la CE-, y que muchos autores reconocen como intransferible e indisponible ${ }^{44}$. Buena fe que se debe hacer tangible en que la arquitectura del sistema de IA o en la formulación del sistema de algoritmo para que sean absolutamente transparentes ${ }^{45}$.

44. En este mismo sentido, Valverde Asencio 2020, 23: "La titularidad del poder de dirección es consustancial a la empresa de conformidad, entre otros argumentos, con los derechos reconocidos, en nuestro país, en el artículo 38 de la Constitución”. Igualmente, Cruz Villalón 2018, 202-203, sosteniendo que - de este poder de dirección-, "deriva su facultad organizativa, en la que precisamente, se incluye la implementación del sistema inteligente".

45. Valverde Asencio 2020, 24: "El carácter relativamente abstracto (del deber de buena fe) debe informar al contrato de trabajo, puede encontrar algunos elementos de concreción en relación con la implantación de los sistemas de inteligencia artificial. Entre estos elementos, primeramente, hay que referir uno básico y elemental. Nos referimos al deber de que dicho sistema sea transparente”. 
Se percibe el interesante debate en torno a si resulta exigible por ley la existencia de una información significativa sobre la "lógica aplicada» al uso de estas herramientas matemáticas, y de como resulta igualmente imprescindible el desarrollo de instrumentos que materialicen el derecho a la transparencia: un auténtico derecho fundamental de la nueva ciudadanía digital, tal y como sostiene algún autor de la doctrina ${ }^{46}$. Debate este -el de la formulación eficaz de la transparencia- que se antoja absolutamente determinante para la consideración del alcance del riesgo de la discriminación ${ }^{47}$.

\subsection{Materialización del riesgo de la discriminación en la utilización concreta de estos sistemas}

El algoritmo necesita de datos. No se trata tanto de que su mecanismo de formulación esté sesgado y, como consecuencia de ello, sus resultados evidencien la existencia de una eventual situación de discriminación. Se trata de que los datos pudieran estar ya sesgados por parámetros discriminatorios. En este sentido el algoritmo se nutre de la realidad como factor de aprendizaje, a resultas de lo cual el resultado de la formulación del algoritmo puede estar contaminado por los sesgos de discriminación que se evidencian en la realidad social del momento ${ }^{48}$. Sobre la base de esta premisa podemos concluir que, el procesamiento automatizado de datos incrementa exponencialmente las posibilidades de vulneración de los derechos de los

46. Mercader Uguina 2021, 7.

47. Del riesgo de estas técnicas es consciente el actual Comité Europeo de Protección de Datos, que, en sus Directrices sobre decisiones individuales automatizadas y elaboración de perfiles a los efectos del Reglamento 2016/679 (WP 251 del GT29), subraya el peligro asociado al uso de estas fórmulas señalando que "la elaboración de perfiles y las decisiones automatizadas pueden plantear riesgos importantes para los derechos y libertades de las personas que requieren unas garantías adecuadas. Estos procesos pueden ser opacos. (...) La elaboración de perfiles puede perpetuar los estereotipos existentes y la segregación social. Asimismo, puede encasillar a una persona en una categoría específica y limitarla a las preferencias que se le sugieren (...). En algunos casos, la elaboración de perfiles puede llevar a (...) una discriminación injustificada".

48. En este mismo sentido, sostiene Todolí Signés 2018, 6, que "la construcción del Algoritmo requiere de datos que están sesgados por parámetros discriminatorios. En efecto, el algoritmo toma la realidad como factor de aprendizaje a la hora de procesa datos, lo que implica que los resultados obtenidos por estos datos vendrían a perpetrar sesgos ya existentes en nuestra sociedad"; (..) "Cuando un algoritmo está al mando, en general, las minorías siempre estarán en desventaja. La propia ciencia de la estadística otorga más valor a las decisiones tomadas con mayor información disponible. Eso conlleva que las minorías -raza, religión, orientación sexual, etc.- habrá menos datos disponibles lo que implicará que el algoritmo entienda que tomar una decisión favorable a un colectivo minoritario sea más arriesgado que tomarla a favor de un colectivo mayoritario. Es decir, que para seleccionar a un candidato de un colectivo minoritario el algoritmo exigirá mas cualidades, aptitudes, conocimientos... (por defecto) que para hacerlo con el de un colectivo mayoritario, simplemente por el hecho que es más fácil predecir -estadísticamente- el comportamiento de un pertenece a este y no a aquel". 
trabajadores ${ }^{49}$. Ello con independencia de que finalmente sea el empresario -a través del responsable de recursos humanos- el que tome una determinada decisión, el mero hecho de que lo haga basado en un procesamiento automatizado de datos provocará un incremento en las probabilidades de que la decisión tomada resulte discriminatoria.

Llegados a este punto, podríamos plantearnos si la formulación del art. 80.5 del Convenio de la Banca resulta eficaz de cara a contrarrestar estos riesgos de discriminación, y si plantea alguna novedad reseñable desde la perspectiva de la gobernanza de la IA en el ámbito laboral. En relación con la primera cuestión recordemos que el precepto obliga a las empresas a «informar a la representación legal de los trabajadores ${ }^{50}$. Observamos por tanto que la participación de la representación de los trabajadores es requerida ante la ausencia de intervención humana en el proceso de toma de decisiones y, que el contenido mínimo que configura el que hemos analizado como derecho a la explicación incluye expresamente «a los datos que nutren a los algoritmos». Elemento que, como hemos observado es susceptible de albergar rincones oscuros o inaccesibles al conocimiento de las personas trabajadoras; por tanto, riesgo potencial de discriminaciones.

Circunstancia esta muy relacionada con la segunda de las cuestiones planteadas desde la perspectiva del modelo de gobernanza del art. 80.5 5 . La novedad radica en la incorporación del derecho del trabajador a solicitar que personas designadas por la empresa intervengan. Sin más detalle y sobre la base de lo expuesto, parece entenderse que, una vez informada a la persona trabajadora del contenido mínimo de su decisión (derecho a la explicación), en caso de discrepancia, y antes de la interposición de una eventual acción de reclamación, la persona trabajadora tiene el derecho a solicitar la participación de personas responsables, -no se trata de persona individual sino más bien alguna comisión-, que se intuye tendrá acceso a la verificación o la trazabilidad del proceso o de la toma de decisión que proceda, comprobando la inexistencia de sesgos de discriminación.

Sin duda la participación de más de una persona que intervenga a posteriori sobre los resultados de los sistemas analíticos- que -hasta ese momento han intervenido de manera autónoma- incorpora un mecanismo que genera fiabilidad y cierta

49. Así, se dirá que Internet implica "el riesgo de un efecto multiplicador de los atentados contra derechos, bienes e intereses jurídicos”: Perez Luño 2006, 93. En el mismo sentido: Garriga Domínguez 2018, 109.

50. Recordemos: "uso de la analítica de datos o los sistemas de IA cuando los procesos de toma de decisiones en materia de recursos humanos y relaciones laborales se basen, exclusivamente en modelos digitales sin intervención humana. Dicha información, como mínimo, abarcará los datos que nutren los algoritmos, la lógica de funcionamiento y la evaluación de los resultados”.

51. Literalmente establece: “...en relación con las decisiones y procesos, cuando ambos estén basados únicamente en algoritmos, pudiendo solicitar, en estos supuestos, el concurso e intervención de las personas designadas a tal efecto por la Empresa, en caso de discrepancia”. 
seguridad jurídica, más allá del contenido mínimo del derecho a la explicación. Sin conocer el alcance concreto de ese concurso o intervención, si lo unimos con el hecho de que la información contendrá como mínimo los datos de los que se nutren los algoritmos, se perciben interesantes indicadores de transparencia que además dotan de mayor concreción el deber de buena fe empresarial en la implementación de estos procesos.

\section{Conclusiones}

Conforme avanza el estado de la tecnología -absolutamente vertiginoso en estos momentos- se multiplican las formas de procesamiento de datos e información. Este hecho repercute inexorablemente sobre: a) la adaptación (y mayor complejidad) de la regulación en materia de protección de datos, en aras a tratar de ser más garantista de los derechos fundamentales de la ciudadanía en general y de las personas trabajadoras en particular y; b) la naturaleza jurídica de los conflictos que surgen al hilo de este proceso. De tal manera que una de las principales consecuencias de la implantación de los algoritmos e IA en las relaciones de trabajo, sería el propio estado de evolución de estas tecnologías ${ }^{52}$. A resultas de lo cual, a mayor complejidad tecnológica, nuevos y más conflictos jurídicos complejos. Así, hemos pasado, en un breve lapso de tiempo, de la preocupación por la privacidad / intimidad de las personas trabajadoras en el puesto de trabajo a la preocupación (legislación) por como esa protección (diseñada para proteger privacidad) puede adaptarse a la protección al derecho a la igualdad y a la no discriminación.

Como quiera que la tecnología es capaz de inferir información sensible -potencialmente discriminatoria- a través de otros datos e información, forma parte de las obligaciones del empresario explicar con detalle cómo se ha alcanzado determinada decisión y porqué. Tal como indica Todolí Signes ${ }^{53}$, se entiende que el incremento de la trasparencia en la toma de decisiones es necesario en un mundo donde la tecnología permite grandes posibilidades de discriminación. Además, una explicación completa de los fundamentos utilizados por la tecnología para tomar determinada decisión se entiende necesaria para evitar la indefensión del afectado y para que esta pueda oponerse -o alegar lo que estime oportuno- ante dicha decisión (art. $24 \mathrm{CE}$ ).

Como hemos tenido ocasión de comprobar, no sólo se trata de proteger los datos de los trabajadores, sino de hacer un buen uso de los mismos cuando son considerados en procesos de toma de decisiones que les afectan directamente. Esto es, articulando instrumentos o diseńando el propio modelo de toma de decisiones, para

52. En el mismo sentido: Instituto Cuatrecasas 2019, 31.

53. 2018, 20. 
evitar el riesgo de vulneración de derechos básicos de las personas trabajadoras. Observamos como el art. 80.5 del Convenio Colectivo de la banca ofrece un interesante enfoque regulatorio incidiendo en aspectos tales como el origen de los datos de los que se nutre el sistema de IA (transparencia) e incorporando el derecho a solicitar el concurso e intervención de personas designadas por la empresa para verificar los resultados del proceso (gobernanza). Ello sin pasar por alto que es la propia negociación colectiva el instrumento más idóneo para ir configurando un proceso garantista, con gran capacidad de adaptación al incesante proceso innovador de las tecnologías. En este sentido, deben ser los representantes legales de los trabajadores, los titulares del derecho de información y explicación sobre los sistemas algorítmicos de contratación o de gestión de personal y a los que la norma debe -reformándose el art. 64 LET-, igualmente, garantizar competencias de participación y control sobre las auditorias algorítmicas.

Vista la incidencia que las tecnologías de la información y comunicación tienen en las relaciones laborales, la empresa debe ser consciente de que tiene ante si, una nueva obligación de dotar de más contenido al deber de buena fe que le resulta inherente en la gestión diaria de las relaciones de trabajo. En este caso, la aplicación de medios tecnológicos introduce elementos novedosos en las relaciones laborales. Las ventajas y consecuencias positivas, en términos de eficacia y eficiencia sobre las condiciones de trabajo de las personas trabajadoras, deben ser perfectamente compatibles con la finalidad del derecho del trabajo en su función reequilibradora de intereses, protectora de la parte más débil contractualmente o de gestión de los recursos humanos y mejora de la productividad de las empresas.

Es este un argumento ya clásico pero de formulación práctica compleja, que obliga a todos los actores con capacidad de decisión en el ámbito de las relaciones laborales, a gestionar el recurrente "mientras tanto". Esto es, mientras se publica una legislación específica en la materia (cristalicen las diversas iniciativas comentadas), que sea la negociación colectiva el instrumento que, como en el caso que nos ocupa, ofrezca soluciones innovadoras garantistas de los derechos fundamentales de las personas trabajadoras. Que termine superando la situación de debilidad manifiesta en un proceso de negociación individual (trabajador-empresario) en materia de protección de datos. Ofreciendo soluciones que incluyan necesariamente: el conocimiento exacto de la trazabilidad de la decisión y la transparencia durante todo el proceso, (en especial sobre los datos que lo nutren), el derecho a obtener por parte de la empresa una explicación completa, comprensible ${ }^{54}$ y evidenciable de los resultados de las decisiones. En definitiva, tratar de aportar asideros de claridad y certeza jurídica a tan complejo asunto.

54. GT29 en Guidelines on Automated individual decision-making and profiling for the purposes of Regulation 2016/679, adoptadas el 3 de octubre de 2017, pág. 25. 
6. Bibliografía

Becerra, Jairo (Ed.); Cotino Hueso, Lorenzo; León, Ivonne Patricia; Sánchez-Acevedo, Marco Emilio; Torres Ávila, Jheison; Velandia Vega, John, (2018), Derecho y big data, Bogotá (Colombia).

Casey, Bryan (2018), “The Next Chapter in the GDPR's "Right to Explanation” Debate and What It Means for Algorithms in Enterprise", European Union Law Working Papers, 29, https://law.stanford.edu/publications/no-29-next-chapter-gdprs-right-explanation-debate-means-algorithms-enterprise/

Cruz Villalón, Jesús (2018), Compendio de Derecho del Trabajo, Madrid.

Documento UGT (2021), Las decisiones algoritmicas en las relaciones laborales. De 8 de febrero de 2021. Enlace a documento completo, acceso con fecha de 13.10.21, en https://servicioestudiosugt.com/las-decisiones-algoritmicas-en-las-relaciones-laborales/.

Evans-Freenwood, Peter; Lewis, Harvey; Guszcza, Jum (2017), "Reconstructing work: Automation, artificial intelligence, and the essential role of humans", Deloitte Review, 21; https:/www2.deloitte.com/us/en/insights/deloitte-review/issue-21/artificial-intelligence-and-the-future-of-work.html

Garriga Domínguez, Ana (2018), "La elaboración de perfiles y su impacto en los Derechos Fundamentales. Una primera aproximación a su regulación en el Reglamento General de Protección de Datos de la Unión Europea”, Derechos y Libertades, 38, 107-139.

Goodman, Bryce; Flaxman, Seth (2016), «EU Regulations on Algorithmic Decision Making and «a Right to an Explanation", AI Magazine, vol. 38, 3, 50-57.

Gobierno de España (2021), Carta de Derechos Digitales. Acceso a documento completo para consulta pública (13.10.21); https://portal.mineco.gob.es/RecursosArticulo/mineco/ministerio/participacion_publica/audiencia/ficheros/ SEDIACartaDerechosDigitales.pdf.

Informe FRA. European Union Agency for Fundamental Rights (2018), \#BigData: Discrimination in data-supported decision making.

Inneratity, Daniel (2020), Una teoría de la democracia compleja: Gobernar en el Siglo XXI, Madrid.

Instituto Cuatrecasas de Estrategia Legal en Recursos Humanos (2019), Informe general. El impacto de las tecnologías disruptivas en la gestión de los Recursos Humanos y en el marco regulatorio de las Relaciones Laborales. Proyecto Technos, Madrid.

Mantelero, Alessandro (2018), "AI and Big Data: A blueprint for a human rights, social and ethical impact assessment", Computer Law and Security Review, 34.

Mencken, Henry Louis (1986), Prejudices: Second Series, Nueva York.

Mercader Uguina, Jesús (2021), "Discriminación algorítmica y derecho granular: nuevos retos para la igualdad en la era del Big Data”, LABOS Revista de 
Derecho del Trabajo y Protección Social, Vol. 2, 2. Doi: https://doi.org/10.20318/ labos.2021.6213

Perez Luño, Antonio Enrique (2006), La tercera Generación de derechos humanos, Cizur Menor (Navarra).

Robles Carrillo, Margarita (2020a), “Artificial Intelligence: From Ethics to Law”, Telecommunications Policy, vol. 44, 6. https://doi.org/10.1016/j.telpol.2020.101937.

Robles Carrillo, Margarita (2020b) "La gobernanza de la Inteligencia Artificial: contexto y parámetros Generales”, Revista Electrónica de Estudios Internacionales (REEI), 39; DOI: 10.17103/reei.39.07.

Sáez Lara, Carmen (2020), "El Algoritmo como protagonista de la relación laboral. Un análisis desde la perspectiva de la prohibición de discriminación”, Temas Laborales, 155.

Todolí Signés, Adrián (2018), “La gobernanza colectiva de la protección de datos en las relaciones laborales: big data, creación de perfiles, decisiones empresariales automatizadas y los derechos colectivos", Revista de Derecho Social, 84, 69-88.

Valverde Asencio, Antonio José (2020), Implantación de sistemas de inteligencia artificial y trabajo, Albacete.

Wachter, Sandra; Mittelstadt, Brent; Floridi, Luciano (2017), "Why a Right to Explanation of Automated Decision-Making Does Not Exist in the General Data Protection Regulation", International Data Privacy Law, 7, 76-99. 